period for the same range of work amounted to about $£ 2.5 \mathrm{~m}$.)

The Protein Chemistry Division is in fact part of the Wool Research Laboratory of the C.S.I.R.O., which has also published its annual report for 1965-66. The laboratory is in three parts; the Protein Chemistry, Textile Physics, and Textile Industry divisions. Total expenditure is about $\$ 2.35 \mathrm{~m}(£ 0.94 \mathrm{~m})$. The work of the laboratory covers all the operations of the wool industry, from the sheep to the finished article, and recently a new resin shrinkproofing technique was announced. The laboratory hopes that it will prove to have some outstanding advantages compared with other processes.

\section{Information, Ebb and Flow}

A PARTICIPANT at the recent symposium on the problems of documentation and automation in the handling of scientific information, organized in London by the Ciba Foundation, writes to say that it is becoming clear that some 80 per cent of the communication between scientists does not take place by means of scientific papers proper. Of the remainder, some flows through more personal channels as shown by the increasing circulation of preprints and the growth of other forms of pseudo-publication. Although the need to publish formally is at the heart of the scientific life, approbation and prestige go to the creator rather than the communicator.

The climate of opinion at the conference also suggested that the English language as the principal medium for scientific communication is no longer as dominant as in the fifties. More consideration must be given to other important scientific languages. Machine techniques are promising. The early euphoria about automatic information retrieval based on a utopia of time-shared computers with gigantic memories and high speed operation, widely accessible from remote terminals, has given place to mild disenchantment. Nevertheless, automatic retrieval is a more hopeful prospect than mechanical translation, where progress does not seem to be keeping up with the burgeoning of language. The most rewarding applications of computer techniques have so far been at the level of storing, arranging and printing out indexes, which can vastly speed up the whole process of transferring information. On the other hand, in data retrieval, achievements can have a revolutionary significance as has already been shown for organic chemistry with the computerized system being operated by the American Chemical Society for the Chemical Compounds Registry.

The truth is that information science is far from being a science-there are few accepted general principles, it is still in the "phlogiston era", as it were. Is natural language indexing as adequate for retrieval as the more expensive controlled vocabularies? What is the trade-off between recall and relevance or can both be improved beyond the present low level ? What do users want or really need and how far should information services aim at high retrieval efficiency in any one subject? In organizational terms, the economic pay-off and governmental responsibility are far from clear. In fact how should central governments finance and co-ordinate information services both internationally and nationally so as to maintain a healthy balance of decentralization ?
These were some of the more distant problems raised at the symposium and not, at this stage, solved. There does, however, seem to be general agreement that if the computer is to be used efficiently there must be international sharing of intellectual input (cataloguing and indexing) and a broad acceptance of standardized formats and classification systems. Above all, there must be very much more experimental evaluation of documentation systems.

The meeting was attended by some 25 participants, most of them professionally concerned with the organization of scientific information either in government establishments, industry or the learned societies.

\section{Tools for Protein Chemists}

\section{by a Correspondent in Molecular Biology}

DURING the past few years a number of reagents for the selective modification of specific residues in proteins have been brought to light and are proving to be of great value for the study of conformation and quaternary structure on the one hand and of active sites of enzymes on the other. The current issue of Biochemistry contains good examples of new selective reagents and their applications. Thus King (Biochemistry, 5, 3454 ; 1966) has found that arginyl residues can be modified by condensation with malonaldehyde under acid conditions. The process is not wholly devoid of side reactions; in particular, the acid catalyses disulphide interchange and leads also to a small degree of peptide bond hydrolysis. A further drawback is that the protein is necessarily denatured under the conditions of the reaction. Several proteins were treated, and high degrees of conversion were achieved. It is interesting that the peptide bond following the arginyl residue is rendered resistant to attack by trypsin, and applications in the determination of sequences suggest themselves.

A reagent devised by Shibata and his associates, diazonium-1H-tetrazole, has been re-examined by Sokolovsky and Vallee (ibid., 3574). They find that histidine and tyrosine residues are attacked to give monoazo- and bisazo-derivatives, and the overlap of the absorption spectra of the modified residues is such that there is some ambiguity between reactive tyrosine and histidine residues. The ambiguity is completely resolved only when all tyrosines and histidines have fully reacted, which requires prior denaturation of the protein. Another selective reagent which modifies tyrosyl residues of proteins is tetranitromethane (Sokolovsky et ab., ibid., 3582). A side reaction is oxidation of cysteine. Nitration of the tyrosine residues again introduces a new chromophore, which is of use in following the reaction, and for which other uses may appear.

A good example of the application of a specific reagent to the study of enzyme function is provided by Pontremoli et al. (ibid., 3568). Tyrosyl residues may be specifically acetylated with the reagent $\mathrm{N}$-acetylimidazole, and the effect of such modification on the enzyme fructose diphosphatase has been studied. It is found that ten tyrosyl residues can be modified. The reaction is time dependent, and if it is stopped at the stage at which only two or three residues are modified, the enzyme remains fully functional. If four further tyrosines are acetylated, the enzyme remains active, but loses the capacity to be inhibited by AMP-a specific non-competitive inhibitor. These 